\title{
Una mirada a las estrategias de difusión y competitividad de las industrias culturales y creativas latinoamericanas
}

\author{
A look at latin american cultural and creative industries' diffusion and \\ competitiveness strategies
}

\author{
Andrea Carolina Redondo Méndez' (iD (:8), Carlos Andrés Pinzón Muñoz²(1D) (8:), \\ Oswaldo Ospina Martínez ${ }^{3}$ (iD (8:)
}

\section{Cómo citar}

Redondo Méndez, A.C., Pinzón Muñoz, C.A. y Ospina Martínez, O. (2021). Una mirada a las estrategias de difusión y competitividad de las industrias culturales y creativas latinoamericanas. Socialium, 5(2), 1-20. https://doi.org/10.26490/uncp.sl.2021.5.2.935

${ }^{1}$ Magister en Investigación Social Interdisciplinar, Politóloga. Universitaria Agustiniana. Bogotá, Colombia. andrea.redondo@uniagustiniana.edu.co

${ }^{2}$ Magíster en Desarrollo sostenible y medio ambiente. Universitaria Agustiniana. Bogotá, Colombia carlos.pinzonn@uniagustiniana.edu.co

${ }^{3}$ Magíster en psicología del consumidor. Administrador de empresas.

Universitaria Agustiniana, Bogotá, Colombia. oswaldo.ospinam@uniagustiniana.edu.co

Arbitrado por pares ciegos

Recibido: 10/04/2021 Aceptado: 28/06/2021

\section{RESUMEN}

El objetivo de la investigación es identificar las estrategias que han utilizado las industrias culturales y creativas de Latinoamérica para la generación de competitividad y los procesos de difusión de sus bienes y servicios, mediante un diseño investigativo no experimental que permitió evidenciar la situación del sector cultural latinoamericano, con metodología cualitativa de acercamiento directo con 50 empresarios del sector creativo y cultural latinoamericano, a través de entrevistas en profundidad; como principal hallazgo se encontró que la estrategia más utilizada, frente a su presencia en el mercado y para el mejoramiento de la competitividad dentro del sector cultural, ha sido el networking de tipo cultural, ya que les ha permitido el reconocimiento y la retroalimentación de quienes mayor experiencia tienen en el sector, así como la capacidad para identificar escenarios de acción y tejer redes humanas de colaboración y conocimiento. Sin embargo, y como conclusión, esta estrategia no ha representado, para el sector, los resultados esperados, al ser de tipo autónomo y no una estructura establecida desde estándares estatales u organizacionales que soporten el sector cultural en cada uno de sus países, faltando así el apoyo del Estado y su reconocimiento como industrias representativas en la región.

Palabras clave: networking; industrias culturales y creativas; competitividad; difusión.

\section{ABSTRACT}

The aim of the research is to identify the strategies that have used the cultural and creative industries of Latin America for the generation of competitiveness and the processes of diffusion of their goods and services, through a nonexperimental research design that showed the situation of the Latin American cultural sector with qualitative methodology of direct approach with 50 entrepreneurs from the Latin American creative and cultural sector through in-depth interviews; as the main finding was found that the most used strategy, given its presence in the market and for the improvement of competitiveness within the cultural sector, has been cultural networking, since it has allowed them to recognize and feedback from those with the most experience in the sector, as well as the ability to identify scenarios for action and weave human networks of collaboration and knowledge. However, and in conclusion, this strategy has not represented, for the sector, the expected results, being of an autonomous type and not a structure established from state or organizational standards that support the cultural sector in each of its countries, without the State's support and its recognition as representative industries in the region are lacking.

Keywords: networking; cultural and creative industries; competitiveness; diffusion. 


\section{Introducción}

Para las industrias culturales y creativas, la generación de competitividad y el logro de reconocimiento social a partir de la difusión y del mejoramiento de procesos y etapas en las cadenas de valor y de creatividad, se ha convertido, en las últimas décadas, en objetivos para el logro del crecimiento del sector cultural; esto, debido a que no existen mecanismos claramente definidos ni estrategias clarificadas que fortalezcan este tipo de actividades en la región. Si bien, se han definido cada vez más, espacios de concentración y de conocimiento para ellas, no se ha logrado establecer estrategias efectivas para su posicionamiento. Razón por la cual, se indaga sobre las estrategias más comunes y eficaces para el logro de mayores niveles de competitividad y de difusión para emprendimientos creativos a partir de su propio accionar y definido desde sus capacidades. Así, el objetivo es identificar las estrategias que han utilizado las industrias culturales y creativas de Latinoamérica para la generación de competitividad y los procesos de difusión de sus bienes y servicios.

El networking, como teoría, ha tenido innumerables desarrollos a partir de sus ventajas en diversas industrias, debido a que se ha presentado como la habilidad que los empresarios necesitan desarrollar para crear oportunidades y para cosechar las ventajas que puede adquirir para sus negocios. (Lindsay, 2005); y como una estrategia de desarrollo que combina el reconocimiento de los actores con una amplia colaboración voluntaria entre diferentes entidades, y la fuerza impulsora como interés común, para desarrollar habilidades, o para aprender (Akbar, 2014).

Como concepto supone, per se, una red humana de trabajo productivo que permite el intercambio de experiencias y el aumento del espectro de oportunidades de negocios incluso, para el sector cultural, ofreciendo acciones estratégicas orientadas al logro de cada uno de los participantes, lo que se traduce en reconocimiento del otro, trabajo colaborativo y conexiones futuras, tan importantes en las redes humanas. Ahora, desde una perspectiva más amplia, se debe decir que el networking

es el arte de crear, gestionar, ampliar y mantener tu red de contactos, vía on-line [virtual: redes sociales, redes profesionales, IM, Blog, Web, etc.] y off-line [presencial: eventos, desayunos, comidas y cenas de negocios, encuentros, etc.] de manera sistemática para que ambas partes ganen, se generen sinergias, colaboraciones, negocios, etc. Consolidando relaciones duraderas y de confianza. (Porras, 2013, p. 6)

Lo que plantea una utilización amplia del concepto dentro de procesos de interdependencia para diversos sectores productivos, al generar acciones colaborativas de niveles variados dentro de una misma iniciativa, creando alianzas duraderas y de beneficios bilaterales. Visto así, la necesidad de que pueden ser establecidas desde tres características que han sido propias de esta estrategia, a saber, el compromiso 
de los miembros frente a las tareas asignadas; la gestión compartida entendida como la participación de todos los miembros en todos los ámbitos de actuación de la red; y el equilibrio frente a los proyectos o propuestas que se generen dentro de la red. Así, se depende de la acción conjunta libre de prejuicios y competencias ya que se trata de un compartir de riesgos y oportunidades con el fin de visibilizar la red y mejorar realidades a través del logro de los objetivos propuestos.

Ahora bien, desde el punto de vista cultural, esta estrategia está enmarcada, no en estándares tradicionales de sectores históricamente reconocidos, sino dentro de parámetros tendientes a la caracterización y conocimiento del grupo de industrias que componen la economía creativa, debido a que supone el establecimiento de estrategias para ingresar al mercado, para actuar en contextos cambiantes y para la difusión de su propio arte en espacios cerrados, de difícil acceso o desconocidos. Y es que se trata de espacios de cooperación ya sea dentro de la misma región o fuera del continente.

Sin embargo, para lograr establecer una relación de su funcionamiento con las Industrias culturales, es necesario reconocer que el desarrollo de los conceptos de competitividad o difusión se encuentra claramente relacionado con la noción de competencia, al incluir dentro de sus estructuras epistemológicas una serie de acciones ligadas a las raíces de lo que significa competir, tanto a nivel individual como a nivel sectorial. Es decir, al hablar de competitividad, las industrias culturales hacen uso de las herramientas del emprendimiento cultural y por ende de las redes humanas, en la medida en que se logra establecer relaciones tanto dentro como fuera de su organización, y así, impulsar alianzas que generen mejores resultados en conjunto, para todo el sector cultural, a través de la inclusión a nuevos mercados.

Como antecedentes investigativos, se debe mencionar, en primer lugar, la guía de la UNESCO (2010b), "políticas para la creatividad" en donde se inicia un recorrido para planificar e implementar una hoja de ruta que ayude al sector cultural a lograr un posicionamiento como industria desde financiamiento hasta la competitividad. Se trata de uno de los primeros textos que vincula, no solo la definición de este sector, sino una serie de pasos y estrategias para lograr unos mayores niveles de desarrollo.

En segundo lugar, se debe mencionar el trabajo que desde el Banco Interamericano de Desarrollo se estructuró, en cabeza de Grazzi y Benevente (2017), para el desarrollo de este sector, "Políticas públicas para la creatividad y la innovación" que pone especial énfasis en el papel institucional para fortalecer la difusión y la competitividad de las industrias culturales y creativas en América Latina.

Por último, se debe mencionar, el libro "Industrias culturales y economía creativa en Latinoamérica: Desarrollo económico y social en la región" (Hernández et al., 2018), quienes presentan un recorrido por los avances latinoamericanos de este sector, y sus posibilidades de crecimiento, así como las necesidades y ejemplos de algunos países de la región. 
A partir de lo anterior, este artículo presenta un acercamiento al concepto de industrias culturales y creativas, una relación con la llamada competitividad cultural, los resultados del instrumento presentado y la discusión que genera a partir de las experiencias, en tanto, la identificación de estrategias dentro de las industrias culturales y creativas de Latinoamérica supone una revisión conceptual inicial que de paso a su entendimiento como parte del sistema económico y como garantes de desarrollo.

\section{Método}

El diseño metodológico aplicado fue no experimental, teniendo en cuenta que se trató de un análisis de situaciones preexistentes en las industrias culturales y creativas de América Latina. Este, implicó abordar, no solo a los empresarios, artistas y conferencistas de 10 países de la región, sino que vinculó variables de interés que permitieron identificar el tipo de estrategia que cada uno utilizaba para su difusión y para el logro de competitividad. La pregunta de investigación que guio el proceso metodológico fue ¿̇uáles son las estrategias que las ICC utilizan en América Latina, para lograr mayor difusión y competitividad?, lo cual se logra responder a partir de los siguientes elementos.

Tipo de estudio. Se utilizó un diseño investigativo no experimental con metodología cualitativa, puesto que se fundamentó en la observación de fenómenos tal y como se dan en su contexto natural para después analizarlos. Mediante el enfoque cualitativo, se logró indagar en la naturaleza profunda de las situaciones que caracterizan al sector cultural en la región latinoamericana, además del sistema de relaciones que presenta al buscar su reconocimiento y el mejoramiento de su competitividad. Y con el método documental, se logró organizar, interpretar y analizar la información obtenida por los participantes.

Población y muestra. La población estuvo determinada por las siguientes características: actores primarios del ecosistema creativo latinoamericano, que hayan tenido participación en eventos de difusión de sus bienes y servicios culturales liderados por sus gobiernos locales, trayectoria de más de 5 años. Los participantes fueron empresarios, artistas y conferencistas pertenecientes a diez países: Argentina, Bolivia, Brasil, Chile, Colombia, Ecuador, Paraguay, Perú, Uruguay y Venezuela de los sectores de: artes escénicas, audiovisual, diseño, editorial, música, animación y videojuegos. Se trató de una muestra representativa de 50 personas, que cumplían con las especificaciones del universo poblacional inicial, siendo un muestreo probabilístico, de tipo simple aleatorio, teniendo en cuenta que se trató de un universo de 5 actores primarios por país, que han participado en eventos culturales de integración en la región. La selección de la muestra tuvo que ver con la unidad de análisis que se utilizó, a saber, 
emprendimientos, iniciativas o grupos formalmente constituidos que han tenido espacios de difusión fuera del territorio de origen y que han logrado un reconocimiento y presencia en el mercado internacional; esto con el fin de identificar el tipo de estrategias que han utilizado para este objetivo.

Instrumentos de recolección de datos. Para la investigación se contó con 50 entrevistas en profundidad a empresarios culturales; igualmente, se tomaron las siguientes variables de interés, con el fin de lograr identificar la existencia, desarrollo y funcionalidad el networking cultural, categorías: subsector cultural, dedicación a la actividad en tiempo, niveles de inserción en el mercado, conocimiento de demás subsectores y niveles de relación con la industria en su país. De la misma manera, se diseñó un guion semiestructurado, el cual se dividió en subcategorías: fuentes de financiación de los proyectos, conocimiento de apoyos públicos o privados y su utilización, estrategias o herramientas que utilizan para obtener beneficios económicos y de difusión o reconocimiento, siendo en total 10 ítems, entre categorías y subcategorías indagadas.

Procedimientos de la recolección de datos. Mediante el instrumento diseñado para la recolección de información, las entrevistas en profundidad realizadas, tuvieron como propósito identificar las estrategias y mecanismos utilizados por representantes del sector cultural latinoamericano en la búsqueda de difusión y competitividad. El proceso denotativo incluyó la grabación y posterior paso a texto de cada uno de los testimonios, en tanto se dieron bajo la forma uno a uno. Igualmente se contó con un protocolo para las entrevistas realizadas, el cual se compartió con cada uno al inicio de la misma; permitiendo un espacio de observaciones participativas relevantes y la preparación para el mejoramiento del proceso de recolección de información.

Aspectos éticos. Los aspectos éticos que se tuvieron en cuenta para el desarrollo de esta investigación y que fueron cuidadosamente tratados y establecidos con cada uno de los participantes en las respuestas de este instrumento, se relacionan con la protección de cada uno de los participantes, tanto desde su papel como individuos sociales, como participantes y actores relevantes del sector cultural latinoamericano.

En primer lugar, se tuvo en cuenta la invasión a la privacidad y la confidencialidad de la información que se brindó. En tanto, desde el inicio de la selección de los 50 participantes, el equipo recolector de la información informó el tipo de investigación, el objetivo y el tratamiento de sus datos. Se debe mencionar que se trata de información anónima, tanto de los individuos como de sus empresas. Igualmente, se tuvo en cuenta el límite del alcance de las preguntas, es decir, no se pasaron los límites morales ni culturales de ningún participante. 
En segundo lugar, el aspecto fue la conservación y la difusión de la información recolectada, en la medida en que se solicitó expresa autorización de los participantes para el uso exclusivamente académico de la misma; quitando cualquier tipo de carga emocional o legal en la entrega y difusión de sus opiniones. También se tuvo en cuenta el rol de cada persona dentro del evento en el que participaban y su posición respecto al mismo (Santi, 2013).

\section{Análisis de datos}

El análisis de datos se realizó bajo la modalidad de contenido, teniendo en cuenta que la información obtenida permitió tabular las opciones presentadas por los participantes y clasificar cada una de las estrategias que manifestaron desde su conocimiento y utilización. A continuación, se presentan los pasos que se realizaron para el análisis de la información, a saber, obtención de la información, revisión y transcripción, organización de los datos según las variables utilizadas, categorización y codificación de la información, análisis y creación de hipótesis y conclusiones.

\section{Resultado}

\section{Industrias culturales y creativas: acercamiento inicial}

El desarrollo teórico de las industrias culturales, ha sido objeto de estudio desde diversos planteamientos y disciplinas que han aportado a su proceso y a su posicionamiento dentro de la literatura, ya sea como generadoras de procesos identitarios o como garantes de valor agregado, pero siempre como protagonistas de procesos transformadores e innovadores de crecimiento y progreso. Razón por la cual, se presenta, aquí, un acercamiento teórico inicial, desde una de las vertientes que más logra aportar al objeto de estudio y que permite un relacionamiento de las variables de análisis y los resultados obtenidos. Se parte, entonces, desde uno de los principales referentes teóricos para el entendimiento de la dimensión social y económica de este sector cultural, intentando vincular su estructura y aporte económico desde la creación de contenido; sin embargo, dejando un poco de lado el debate sobre su legado o motivación en su génesis. Por el contrario, demostrando que el estudio de este tipo de industrias estuvo presente en el imaginario académico e intelectual, desde el momento en que se generó un reconocimiento de su multifuncionalidad.

Con Theodor Adorno, las industrias culturales se establecieron como garantes de un espacio individual, personal y subjetivo de esparcimiento o recreación, haciendo referencia a que "toda la praxis de la industria cultural aplica decididamente la motivación del beneficio a los productos autónomos del 
espíritu" (Adorno, 1968). Aludiendo a dos conceptos que eran ajenos y que suponían una reestructuración del pensamiento hacia la comunicación, en tanto se presentaban como conglomerados organizados y cargados de contenido cultural relevante para la identificación y desarrollo de la sociedad, pero también como catalizadoras de demanda, sin importar su libre acceso, ya que vinculan procesos intermedios que generan valor; ya sea desde su publicidad o desde su distribución, lo que produce una lógica económica basada en obtención de capital.

Desde este punto de vista, la definición de las industrias culturales se ve reflejada en su contenido económico y de masificación, tanto de la producción como del consumo, dejando de lado la particularidad y la multiculturalidad propias del sector. Esto es, se pierde la categorización de la habilidad o el talento de los empresarios y emprendedores culturales a ser vistos como un producto más presente en el mercado. Sin embargo, en la actualidad, referirse a este tipo de industrias supone la ampliación del espectro original, debido a que mezcla lo económico, lo cultural y la formalización empresarial en un éxito inagotable. Según la Organización de las Naciones Unidas para la Educación, la Ciencia y la Cultura (UNESCO, 2010a) las industrias culturales son las que "combinan la creación, producción y comercialización de contenidos que son abstractos y de índole cultural". Así, se agrupan dentro de las actividades que producen bienes y servicios asociados con la generación de riqueza desde el aprovechamiento de habilidades y talentos basados en la creatividad, la identidad y el conocimiento. $\mathrm{Y}$ aunque las diversas definiciones estén cargadas de variaciones y elementos según la fuente, lo que es cierto es que el mundo está ante una generación de nueva producción económica en crecimiento que dispone de la cultura como capital que demuestra una riqueza inagotable, pero que necesita de orientación y desarrollo para la consecución de sus objetivos económicos y para el logro de un reconocimiento social representativo. Las industrias culturales y creativas y sus representantes requieren de herramientas que les den la visibilidad necesaria y el reconocimiento de su aporte al crecimiento socio - económico de cada nación.

Para el caso latinoamericano, las industrias culturales

Las industrias culturales y creativas han permitido el desarrollo de iniciativas
empresariales, que no solo se caracterizan por ser innovadoras sino porque
vinculan espacios sociales y económicos que están en una constante búsqueda de espacios
de reconocimiento y difusión, debido a la multiplicidad de subsectores que vincula y a su vez
debido a las actividades que integra. (Redondo, et al., 2020, p. 89)

Así, han logrado posicionarse en el mercado internacional, mediante el desarrollo y uso de sus propias habilidades, manifestando su capacidad de difundir su saber y de generar procesos que le permitan 
aumentar su competitividad. Sea de manera informal como actores privados e independientes o como conglomerados dependientes del Estado.

\section{Competitividad cultural: acercamiento conceptual.}

Desde el enfoque sistémico de Esser (1996) existen dos componentes que definen de manera específica el concepto de competitividad, "el primero es la distinción entre cuatro niveles analíticos (meta, macro, meso y micro), (...); el segundo es la vinculación de elementos pertenecientes a la economía industrial, a la teoría de la innovación y a la sociología industrial” (CEPAL, 1996, p. 40). La competitividad, así, puede generar una transformación de la sociedad desde la integración social mediante la implementación de elementos económicos, tecnológicos y políticos, a través de estos cuatro niveles que reflejan el nivel de competitividad de un sector o industria y su relación con el entorno.

El nivel meta se refiere a la influencia de la organización política y la competitividad industrial en términos de desarrollo económico. Según la Comisión Económica para América Latina y el Caribe (CEPAL, 1996), dentro de este nivel aparecen elementos sociales, culturales, habilidades de organización política y estrategias de integración de los actores.

El nivel macro, incluye características macroeconómicas, políticas cambiarias, fiscales y comerciales en donde intervienen los principales organismos del régimen político de un Estado. El nivel meso establece la importancia de las relaciones entre los diferentes niveles políticos y territoriales del Estado, generando un soporte real a las empresas en términos de políticas tecnológicas, ambientales, de infraestructura y de educación. En el nivel micro, se encuentra la capacidad empresarial respecto a la interacción que logre a nivel estratégico en la gestión de estrategia empresarial, de innovación, producción y de relación con proveedores y productores.

Empero, esta competitividad sistémica resulta inconclusa para las industrias culturales y creativas, en la medida en que no vincula en su definición la idea de trabajo multidireccional integrado, sino que plantea la necesaria espera unidireccional funcional desde los grandes niveles para el sector específico. Se debe tener en cuenta la multiplicidad que posee la cultura para el entendimiento y completamiento de la competitividad, en tanto esta, permite entender un sector y un grupo de industrias cuya lógica de creación de valor difiere de los sectores tradicionales, debido a que,

la cultura sería la forma del sentido que se reproduce en la recursividad de la comunicación social, la cual siempre puede ser diferente de lo que es. La cultura aparece entonces como una comparación en la cual operan correspondencias de lógica trivalente. Esto en el sentido de que ella puede establecerse como un meta- nivel que 
sirve como punto referencia que a pesar de diferencias extremadamente grandes pueda reconocer todavía lo semejante. (Zamorano, et al., 2014, p. 432)

Teniendo en cuenta lo anterior, la competitividad cultural se determina como la proyección social que nace con el establecimiento de apoyo y reconocimiento social mutuo, que no solo se nutre de los apoyos desde niveles Meta o Macro, sino que hace uso del trabajo en redes humanas. Igualmente, depende del networking que se logre establecer al proporcionar un mejoramiento, tanto de la cadena de valor como de los recursos de innovación y creatividad, que son identificadas como variables para el logro de la competitividad y concebidas como "la capacidad de realizar una búsqueda sistemática de oportunidades y soluciones de problemas a través de maneras diferentes de pensar y de actuar, que suelen materializarse en productos y servicios nuevos que satisfacen las necesidades de un público objetivo" (Ministerio de Cultura, 2003, p. 1)

Por otra parte, los niveles de competitividad cultural están relacionados con los atributos personales del empresario, en este caso, acudiendo a las herramientas que plantea esta área de estudio, se trata de liderazgo, asertividad, iniciativa, sentido crítico, creatividad y responsabilidad, entre los más importantes. Así,

el emprendedor se desenvuelve en un espacio económico- social, esto se debe a la interconexión entre su contexto interno (factores individuales, personales) y externo (factores del entorno, con textuales). De esta manera, reúne una serie de habilidades, cualidades, capacidades psíquicas e intelectuales que interactúan para establecer un equilibrio en el desempeño exitoso de sus actividades. (Herrera y Gutiérrez, 2014, p. 289)

Ahora bien, la difusión, a la que se hace referencia se establece como condición que se genera a partir del desarrollo de la identidad personal en conjunto y que adquiere un valor social. Y es esta característica la que permite que se identifique la vinculación de la competencia con el sector cultural, ya que al brindarle la condición de creador de identidad se obtiene un elemento diferenciador que entra a determinar su reconocimiento y su papel unitario dentro del contexto internacional.

Así, hablar de reconocimiento social, es hablar de particularidades y funcionalidades propias de un sector que logra acrecentar su influencia desde su facilidad de ingreso, sus posibilidades de visibilización y su capacidad de transformación del espacio social y de las condiciones económicas de sus protagonistas. Sin embargo, se reconoce a la identidad como factor de referencia para su visibilidad, es decir, se trata de vincular a la identidad social como un elemento que pretende la obtención de reconocimiento, planteándolo así, como un componente de carácter positivo. Entonces, la integración de habilidades humanas desde lo social, para el logro de la competitividad, rompiendo con esquemas tradicionales de 
jerarquías, lo que convierte este escenario en un espacio de diálogo elaborado que presenta interese comunes y el objetivo de crecimiento del sector. El crecimiento está pensado en conjunto, mediante el conglomerado de protagonistas y, en esencia, su reconocimiento y éxito económico.

A partir de lo anterior, la funcionalidad del trabajo en redes humanas para las industrias culturales y creativas se ve reflejada en el éxito del trabajo conjunto mediante el conocimiento y utilización del networking cultural, al establecer una clase de marketing relacional al construir fuertes conexiones que generen beneficios y ventajas reales en los mercados potenciales. Sin embargo, no se trata de una actividad desvinculada o al azar, sino de una actividad que, dentro de la economía creativa, representa una base habitual al momento de ingresar al mercado cultural.

Razón por la cual, los empresarios del sector, al buscar mayores niveles de competitividad deben desarrollar prácticas pro activas al momento de establecer relaciones y canales de comunicación con quienes le permitan dar a conocer su bien o servicio y a su vez financiar sus actividades, en la medida en que, crean los vínculos necesarios para generar ventajas compartidas. A partir de los resultados de la aplicación del instrumento, se encontraron, como comunes a los entrevistados:
a. acceder a nuevos espacios de difusión
b. búsqueda de nueva y efectiva información
c. buenas prácticas del trabajo en equipo
d. retroalimentación entre la red
e. promoción de sus productos y servicios
f. búsqueda de nuevos mercados a través de experiencias de terceros

\section{Figura 1}

\section{Prácticas del sector cultural latinoamericano}

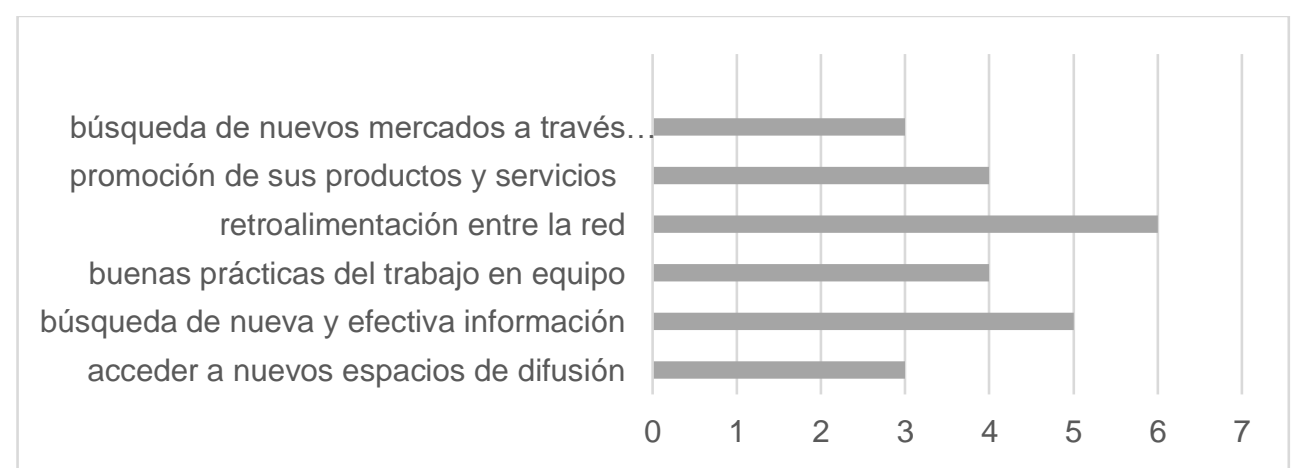




\section{Desarrollo lógico del tema: aplicación del instrumento}

A continuación, se muestran las estrategias, las herramientas y las fuentes de financiación encontradas con la aplicación del instrumento, que además permitieron el enlace y la estructuración desde la teoría hasta la aplicación de la estrategia del networking.

\section{Tabla 1}

Estrategias, herramientas y financiación - empresarios del sector cultural

\begin{tabular}{lcccc}
\hline \multicolumn{1}{c}{ Sector } & Habilidad & Financiación & $\begin{array}{c}\text { Estrategias (difusión - inclusión en } \\
\text { mercados internacionales) }\end{array}$ & Cantidad \\
\hline Audiovisual & Pensamiento sistémico & estatal & Networking & 10 \\
Artes Escénicas & Pensamiento sistémico & estatal & Networking & 15 \\
Audiovisual & Creatividad & privada & Networking & 2 \\
Diseño & Identidad corporativa & privada & Networking & 4 \\
Editorial & Identidad corporativa & estatal & Formación & 2 \\
Música & Innovación & estatal & Networking & 10 \\
Animación & Identidad visual & privada & Comunicación asertiva & 5 \\
\hline Videojuegos & Identidad visual & privada & Inteligencia social & 2 \\
\hline
\end{tabular}

Los resultados frente a las estrategias, herramientas y financiación que se obtuvieron, dan cuenta de una categorización de factores pertenecientes a las industrias culturales y creativas de manera global, que desde el Ministerio de Cultura de Colombia, el Servicio Nacional de Aprendizaje (SENA) y la Universidad de Antioquia se tienen, en tanto se presentan como habilidades y competencias que deben existir dentro del sector cultural por parte de los empresarios para el mejoramiento del desarrollo de su actividad.

Estas habilidades y competencias se definieron como comunicación asertiva, pensamiento sistémico, creatividad y orientación al logro; cada una de ellas delimitando acciones para los protagonistas del sector cultural, poniendo en contexto la manera como deben realizar su gestión y las estrategias que pueden utilizar en cada proceso. Se trató de la búsqueda de diversas fuentes de creación, apropiación financiación y difusión, es decir, nuevas ideas de emprendimiento que generan espacios propios de conocimiento y aprovechamiento de los elementos que son poco resaltados o resaltados de manera insuficiente y equivocada.

$Y$ aunque los resultados no se manifiestan como ajenos al mismo sector cultural, si manifiestan la importancia de un trabajo mancomunado que parte de sus propias habilidades y que se desarrolla a partir de su reconocimiento como sector innovador, al vincular prácticas propias de sus representantes, que 
demuestra la utilidad de la conjunción de habilidades y mecanismos, y que permita la consecución de mejores resultados.

Este conjunto de habilidades y competencias permiten un mayor desarrollo de actividades de internacionalización, generación de competitividad y de visibilidad, integran capacidades y destrezas hasta configurar, desde su estructura, las estrategias garantes de inserción en nuevos escenarios de difusión y de competitividad.

\section{Tabla 2}

Habilidades y competencias del emprendedor cultural

\begin{tabular}{ll}
\hline \multicolumn{1}{c}{ Habilidad/Competencia } & \multicolumn{1}{c}{ Características } \\
\hline Comunicación asertiva & $\begin{array}{l}\text { Habilidad para expresar clara y apropiadamente al entorno actual lo que se siente, piensa y } \\
\text { requiere de forma tal que la transmisión sea fluida y alcance el objetivo propuesto. }\end{array}$ \\
\hline Pensamiento sistémico & $\begin{array}{l}\text { Elaboración de planes de trabajo que establezcan los diferentes elementos como un todo, } \\
\text { relacionándolos al reconocer su favorabilidad en tanto sean capaces de adaptarse a un } \\
\text { escenario cambiante. }\end{array}$ \\
\hline Creatividad & Soluciones novedosas que tienen en cuenta las necesidades del contexto. \\
\hline Orientación al logro & Direccionalidad de las acciones emprendidas hacia los resultados esperados y la constancia en \\
& los mismo.
\end{tabular}

Dentro de los resultados obtenidos, el uso del networking cultural como estrategia principal de los empresarios latinoamericanos para la consecución de mayores niveles de competitividad y para una efectiva difusión, ha sido el elemento más representativo, debido a la funcionalidad que les ofrece no solo de manera formal y organizada, sino que, les ha dado la posibilidad de generar espacios a partir de aglomeraciones del sector desde la informalidad. Es decir, las facilidades de dicha estrategia se ven reflejadas en el desarrollo de cada una de las habilidades presentadas.

Por esto, "la contribución fundamental del intercambio cultural en la economía radica en las experiencias subjetivas de consumo de los consumidores en una sociedad donde las dinámicas tradicionales / institucionales hacen cumplir valores y estilos de vida ideales en los individuos" (Jafaria, 2013, p. 12).

Según los entrevistados, sin importar el sub - sector al cual pertenezca, el establecer redes de trabajo colaborativo mejora las probabilidades de impactar en mercados externos y en lograr mayor visibilidad, debido a que dentro de la conjunción de la generación de valor agregado existen mayores ganancias al momento de vincular a sus copartidarios. 
Igualmente, para el reconocimiento de la estructura humana en redes de las industrias culturales, se logró identificar su origen desde la visión y el trabajo del emprendimiento cultural, debido a la conexión que esto supuso. Es decir, este tipo de emprendimiento presenta la multiplicidad de oportunidades que se generan para las industrias culturales, a partir de las acciones estratégicas basadas en la cooperación.

De acuerdo a la información recolectada, a través del networking cultural los miembros de la red adquieren una multidimensionalidad expresada en las labores que logran cumplir, al establecerse como potenciales clientes y reales proveedores, no solo como consumidores sino como productores de cada una de las etapas de las cadenas de valor y de creatividad. Es así que la dinámica se convierte en un constante escenario de oferta y demanda, con la transversalidad de su cumplimiento basado en esta dualidad. Según los empresarios culturales chilenos del sub sector audiovisual, se trata de la libertad de integrar grupos de trabajo o cooperar con eventos de difusión y promoción de las industrias culturales a través de lazos de ayuda mutua que les permitan darse a conocer en la multiplicidad del mercado creativo. Igualmente, se manifiesta que, al momento de construir una red humana dentro del sector cultural, se invalidan las formalidades y rigideces del propio sistema económico, es decir, vincula al sector por completo en tanto su desarrollo está determinado por la apropiación identitaria y el reconocimiento social que tengan las industrias culturales y creativas, dejando de lado jerarquizaciones empresariales o sociales tradicionales.

Así, se habla de una red autosostenible, no solo respecto a la gestión sino también frente a la financiación, en tanto los niveles de credibilidad y de confianza que hacen parte de su funcionamiento original garantizan su funcionalidad. Sin embargo, se evidenció una de las debilidades más relevantes que se identificó en las redes humanas y es la falta de preparación para las actividades que van surgiendo y que se supone se espera que generen beneficios, sociales, de reconocimiento y económicos para sus participantes.

\section{Discusión}

Desde el reconocimiento de las industrias culturales y creativas de la región latinoamericana, como garantes de crecimiento económico se ha empezado a reconocer su uso del contenido inmaterial, como punto de partida para la creación de valor y de referencia, debido a que permite la apropiación de características innatas y únicas que, además, se muestran en un mercado internacional carente de la identificación real de las identidades de la región, generando así en la sociedad, la identificación y posicionamiento como defensor del patrimonio cultural de la Nación. 
Por esto, el networking que se aplica a las industrias culturales y creativas genera espacios de implementación de características propias del emprendimiento cultural que a su vez forja redes de competitividad basadas en las relaciones humanas y en las particularidades o especialidades de los seres humanos y no a la simple competencia unidimensional, ya que demuestra la multiplicidad de espacios para el logro de mejores resultados, mayor difusión y mejoramiento de la competitividad.

Este trabajo en redes humanas se ha convertido en un paso obligado para cualquier representante del sector cultural, ya que se manifiesta como base de la estructura económica de este tipo de industrias. Recordando que estas se orientan al mercado, un mercado cambiante y en ocasiones incierto para productos creativos, en donde se tiene que trabajar sinérgicamente para dar a conocer el bien o el servicio cultural, de manera tal que se empiece a fortalecer cada subsector. Es por esto que, el estudio del networking desde el sector cultural, lo sitúa como estrategia, no solo de difusión sino de mejoramiento de las capacidades y posibilidades de inclusión en nuevos mercados y así de mejoramiento de competitividad, para una industria que se encuentra en constante crecimiento y que demuestra su aporte al desarrollo económico de la región.

Es importante establecer que la estrategia del networking cultural no actúa en el corto plazo, ya que se trata de un mecanismo que permite una conexión entre protagonistas de la industria cultural y creativa, en donde la integración de todas las esferas va a representar el éxito. Es decir, se trata de todo un proceso de reconocimiento y retroalimentación conjunta, que funciona en la medida en que se presenten como productores en su totalidad y no como estáticos en su rol inicial, de manera que cada una de las esferas logre descubrirse en el otro y pretenda su crecimiento.

Ahora bien, como estrategia de difusión, permite una clara presentación ya que se da en los círculos específicos de accionar cultural, sin embargo, y de acuerdo a los resultados encontrados, la integración en múltiples niveles da la posibilidad de asociatividad siempre que la estructura no se jerárquica, aunque si participativa y democrática. Se trata entonces de lograr una interconexión latinoamericana, que además de generar mayores niveles de competitividad, alejados de la tradicional competencia, va a facilitar la identificación de la región como garante de identidad cultural y como productor directo de las industrias culturales y creativas del mundo.

También, como estrategia de competitividad, el networking cultural demuestra su faceta como generador de espacios de integración comercial novedoso y alejado de estándares tradicionales en donde se compite a través de los espacios que se ganen en la industria y a través de la presencia en nuevos mercados. Demostrando las fortalezas en términos de vinculación y apoyo de las esferas más altas hasta llegar a la manifestación de las propias capacidades del emprendedor creativo. 
De esta manera, se puede convertir en el mecanismo ideal para dar a conocer el valor tangible e intangible de las industrias culturales y creativas de Latinoamérica, siendo estas, motor de crecimiento social y económico, ya que no se debe dejar de lado su papel representativo de la historia latina, ni su relevancia en la comprensión de escenarios de integración, reconciliación e innovación. Por tanto, aporta la estructura esencial en cualquier etapa de la cadena de valor del sector cultural, en tanto vincula y conecta tanto al recurso humano, el recurso económico, como el espacio de difusión.

Así, la reflexión gira en torno a darle la relevancia necesaria al networking cultural como catalizador de apropiación y pertenencia y como integrador lejos de competencias a un sector naciente y en desarrollo para la región latinoamericana, para así, convertirse en un referente para futuras investigaciones que, además, son necesarias para generar un mayor crecimiento en los empresarios creativos. Del mismo modo, para posicionarlo como una herramienta para los nacientes creadores y artistas que no cuentan con todo tipo de recursos para desarrollarse y darse a conocer, debido a que no exige mayores niveles de inversión, ya que tiene su génesis en el propio emprendimiento cultural y en las habilidades propias de sus protagonistas.

La competitividad de las industrias culturales está determinada por la capacidad de sus propios representantes para identificar escenarios de acción y para tejer redes humanas de colaboración y conocimiento, al generar mayores oportunidades de difusión y de reconocimiento social. Esto es, el incremento de la competitividad está directamente relacionado con la capacidad de interrelacionar niveles de accionar desde el Estado hasta la iniciativa privada y así fusionar espacios a través del establecimiento de canales efectivos de comunicación que van a suponer la integración de los actores y su aporte para el aumento de la competitividad. Al hablar de la competitividad cultural, se hace referencia al trabajo integrado y dirigido en múltiples direcciones en tanto se identificó su relación con todos los actores del sector cultural como generadores de procesos de mejoramiento, tanto a nivel público como privado, en la medida en que se teje un trabajo desde la participación de todos los actores, manifestando su carácter colaborativo y completamente alejado de la visión paternalista del Estado como protector y proveedor de cualquier tipo de oportunidad.

Entonces, la discusión que se logra establecer a partir del acercamiento teórico mencionado, tiene su génesis en la caracterización del networking como estrategia fundamental, que además puede ser utilizada en cualquier tipo de industria. Sin embargo, desde el punto de vista cultural, se utiliza como catalizador de oportunidades que van desde la difusión hasta la internacionalización, y que integra los niveles de meta o macro. Así, se presenta como estrategia de competitividad, vinculada a un trabajo en redes, en tanto permite procesos, no solo de generación de riqueza sino de internacionalización, a partir 
del desarrollo de habilidades propias del emprendedor y del establecimiento de espacios en donde confluyen experiencias y procesos diferenciadores de representantes del mismo sector.

\section{Conclusión}

Con el desarrollo de esta investigación, se logra generar un aporte significativo al conocimiento del sector cultura, en tanto se pudo establecer a las Industrias culturales y creativas como garantes de identidad y valor cultural, ya que se basan en la producción de bienes y servicios enfocados en la producción de riqueza desde estándares de identidad cultural que resulta inagotable y se convierte en una de sus más importantes fortalezas, al sobrepasar sectores tradicionales de la economía frente a su contribución al PIB; todo esto, evidenciando la necesidad e importancia del estudio de estrategias e instrumentos que permitan un mayor desarrollo del sector cultural, no solo desde estándares económicos sino desde escenarios de identidad y de representatividad a nivel mundial.

El desarrollo de estrategias, por parte de las industrias culturales y creativas ha sido uno de los temas de mayor relevancia para el impulso del sector cultural en Latinoamérica, debido a que no poseen la estructura necesaria y los lineamientos precisos para la generación de competitividad, actúan de manera empírica, en la mayoría de ocasiones, y los espacios generados son aprovechados por aquellos que poseen mayor experiencia, dejando de lado a quienes inician un emprendimiento y generan ideas cargadas de valor. Así, se ha

empezado a reconocer la unión y el trabajo conjunto entre cultura y comercio, dado que es un aspecto fundamental para el desarrollo de sus economías, ya que no solo se trata de una aseveración de aspectos identitarios resaltados, sino de la utilización de la cultura como catalizadora de emprendimientos, receptora de empleabilidad, de inclusión social y de recepción de inversión. (Falkenstein-Ávila, et al., 2020, p. 3)

Razón por la cual, el networking aparece como estrategia, efectiva, de difusión y de generación de competitividad para el sector cultural, que además no discrimina sobre el tamaño o la experiencia, sino que se basa en las habilidades de los protagonistas del emprendimiento, y en su capacidad de creación de redes colaborativas de trabajo.

A través del estudio realizado se pudo identificar la funcionalidad del networking dentro de las industrias culturales como garante de difusión y de competitividad desde su posicionamiento como estrategia utilizada por empresarios creativos de la región latinoamericana; esto, debido a la identificación de oportunidades de crecimiento para el sector cultural, generadas a partir del desarrollo de habilidades 
comunicativas y el establecimiento de redes. Mediante el desarrollo y aplicación del instrumento utilizado, se logró establecer la relación actitudinal del empresario creativo con la consecución de sus objetivos dentro de nuevos mercados y su accionar direccionado al mejoramiento de su promoción y comercialización.

De la misma manera, se identificó al networking como la estrategia más utilizada frente a la potencialización de su difusión y presencia en el mercado, así como mecanismo de mejoramiento de la competitividad dentro del sector cultural, en tanto permitió un reconocimiento inicial de las iniciativas y los emprendimientos presentados y la retroalimentación de quienes mayor experiencia presentaban dentro del evento; reflejando la cadena de comunicación, como parte de la estrategia, presente en los procesos de networking que se realizaron durante el evento en mención.

Así mismo, se pudo establecer que los tipos de financiación que poseen las iniciativas culturales en Latinoamérica, no determinan el éxito del trabajo en redes ni su efectividad, específicamente, a razón del conocimiento de la estrategia, es decir, el tener o no diversas fuentes de financiación no supone una significación en el éxito de la creación de la red de trabajo y de conocimiento, en tanto la red no necesita una base de capital por cada participante, sino que, por el contrario, se nutre de los talentos y las habilidades que cada uno, de sus conocimientos y sus propias conexiones, que dentro su nivel, puede aportar. Lo que establece el valor agregado de las industrias culturales y creativas respecto a lo inagotable de su materia prima y su posible vinculación a diversas esferas de acción como garantes tanto de innovación y creatividad, como de sentido de pertenencia e identidad.

Cabe resaltar, que, si bien el aporte teórico sobre networking ha sido desarrollado desde diversos puntos de vista, no existe un acercamiento directo desde las industrias culturales y creativas que permita su clara apropiación y utilización, y que la aplicación que se ha realizado responde a intentos empíricos y hábiles de quienes conforman el ámbito cultural en Latinoamérica. Razón por la cual se hace necesaria la instauración de espacios y herramientas para la continuidad en la investigación del networking cultural. Igualmente, se resalta la utilización y desarrollo de las habilidades de los representantes del sector cultural, a saber, comunicación asertiva, creatividad, pensamiento sistémico y orientación al logro, como catalizadores de nuevas oportunidades de ingres a nuevos mercados y la obtención de mejores resultados frente a su propio accionar. Se destaca el trabajo de las redes humanas como generadoras de valor en las industrias culturales, al establecerse como base estructural del crecimiento social y económico del sector, de manera que fortalece la composición bajo la cual se crean y establece una dinámica de relación entre ellas y su entorno caracterizada por su diligencia, productividad y eficiencia al momento de contactarse con sus pares. Siendo el networking el espacio adecuado para generar un mayor crecimiento de la 
industria cultural en Latinoamérica a través de la multiplicidad de oportunidades que logra manifestar desde su creación hasta su comercialización.

Finalmente, es importante decir que esta investigación cobra especial relevancia debido a que no existen mayores desarrollos investigativos y reflexivos frente al tema en la región latinoamericana, y porque, mediante la indagación y la muestra recolectada se evidencia la necesidad de su conocimiento y adecuada utilización, debido a que si bien es un tema nuevo y de fácil acceso, no presenta las suficientes condiciones para su aplicación, entonces se habla de un tema que debe ser en efecto clarificado para obtener los resultados esperados. Esta investigación abre el espectro investigativo para futuras ideas generadas desde la academia y la vinculación con el sector empresarial y así lograr mayor desarrollo para la totalidad del sector cultural de la región.

\section{Referencias}

Adorno, T. (1987). La industria cultural. Santillana.

Akbar, K. (2014). Networking for regional development: a case study. EuroMed Journal of Business, (9), 149-163. https://www.sciencegate.app/document/10.1108/emjb-10-2013-0045

CEPAL. (1996). Competitividad sistémica: nuevo desafío para las empresas y la política. Revista de la Cepal. 59. https://www.cepal.org/es/publicaciones/37977-revista-la-cepal-no59

Esser, K., Wolfgang H. y Meyer-Stamer J. (1996). Competitividad sistémica: Nuevo desafío a las empresas y a la política. Revista de la CEPAL, 59, 32 - 52.

https://repositorio.cepal.org/handle/11362/12025?show=full\&locale-attribute=en

Falkenstein-Ávila, H., Redondo-Méndez, A. y Pinzón-Muñoz, C. (2020). Desarrollo de la economía creativa en Argentina: ejemplo para el caso colombiano. Revista Escuela de Administración de Negocios, Ee 2020, 1-30 https://doi.org/10.21158/01208160.n0.2020.2753

Grazzi, M. y Benavente, J. (2017). Políticas públicas para la creatividad y la innovación: Impulsando la economía naranja en América Latina y el Caribe. Banco Interamericano de Desarrollo. https://publications.iadb.org/publications/spanish/document/Pol\%C3\%ADticas-p\%C3\%BAblicaspara-la-creatividad-y-la-innovaci\%C3\%B3n-Impulsando-la-econom\%C3\%ADa-naranja-en-

Am\%C3\%A9rica-Latina-y-el-Caribe.pdf 
Hernández, J., Redondo A. y Ospina, O. (2018). Industrias culturales y economía creativa en Latinoamérica. Desarrollo económico y social en la región. Editorial Uniagustiniana. https://doi.org/10.28970/9789585498143

Herrera, K. y Gutiérrez, J. (2014). Emprendimiento como iniciativa para la creación de empresa: análisis desde la perspectiva psicológica y conceptual. Revista de Ciencias Sociales. Facultad de Ciencias Económicas y sociales de la Universidad de Zulia, 20(2), 288-302. https://dialnet.unirioja.es/servlet/articulo?codigo $=5196803$

Jafaria, A. (2013). La globalización, la reflexividad, y el proyecto del auto: un proceso de aprendizaje intercultural virtuales. Taylor and Francis Group content.

Lindsay, D. (2005). Cracking the Networking CODE (2da ed.). World Gumbo, Plano, TX.

Mincultura. (2003). Impacto económico de las industrias culturales en Colombia. Reporte técnico. http://www.sinic.gov.co/SINIC/CuentaSatelite/CuentasateliteDocumentos.aspx?CUEID=16.

Ministerio de Cultura. (2009). Política para el emprendimiento y las industrias culturales de Colombia.

Colombia. https://www.mincultura.gov.co/ministerio/politicas-culturales/politicaemprendimiento-industrias-

culturales/Documents/13_politica_emprendimiento_industrias_culturales.pdf

Porras, A. (2013). NET...QUÉ?! Networking para todos. Centro de Estudios Financieros.

Redondo, A., Pinzón, C., y Ospina, O. (2020). Internacionalización de emprendimientos culturales de Latinoamérica. PODIUM, (38), 87-104. https://doi.org/10.31095/podium.2020.38.6

Santi, M. (2013). Hacia un abordaje particularizado de los problemas éticos de las investigaciones sociales. [tesis de maestría]. Facultad Latinoamericana de Ciencias Sociales (FLACSO). https://repositorio.flacsoandes.edu.ec/xmlui/handle/10469/5927

UNESCO. (2010a). Marco de Estadísticas Culturales (MEC) de la UNESCO, sector de la Cultura /Instituto de Estadística [Archivo PDF]. http://unesdoc.unesco.org/images/0019/001910/191063s.pdf

UNESCO. (2010b). Políticas para la creatividad. Guía para el desarrollo de las industrias culturales y creativas [Archivo PDF]. https://es.unesco.org/creativity/sites/creativity/files/220384s.pdf

Zamorano, R., Carrasco, R. y Mendoza, A. (2014). El concepto de cultura en la sociedad moderna: una observación desde la teoría de sistemas sociales. Revista de Ciencias Sociales, (3), 432. https://www.redalyc.org/articulo.oa?id=28032296002 


\section{Contribución de los autores}

ACRM: Conceptualización, metodología, investigación, validación, redacción: revisión y edición. CAPM: Conceptualización, análisis formal, investigación, redacción: borrador original.

OOM: Conceptualización, análisis formal, investigación, redacción: borrador original.

Fuentes de financiamiento

Universitaria Agustiniana.

\section{Conflictos de interés}

No presenta conflicto de intereses.

Correspondencia

andrea.redondo@uniagustiniana.edu.co 